\title{
PAPERS
}

\section{Helicobacter pylori infection does not reduce the viscosity of human gastric mucus gel}

\author{
D C Markesich, B S Anand, G M Lew, D Y Graham
}

\begin{abstract}
The mechanism by which Helicobacter pylori undermines host defence mechanisms is unclear. Several in vitro studies using soluble mucins have suggested that $H$ pylori may compromise mucus function. Gastric mucus gel was obtained from $13 \mathrm{H}$ pylori infected patients; six untreated subjects and seven after eradication of the infection. Gastric mucus is a nonNewtonian substance in that its viscosity changes with changing rates of shear, requiring mucus viscosity to be measured in a rotational cone-plate microviscometer. Viscosity was measured at shear rates varying from $1 \cdot 15 \mathrm{~s}^{-1}$ to $46 \mathrm{~s}^{-1}$. The gastric mucus viscosity was significantly higher in patients infected with $H$ pylori compared with mucus gel obtained after eradication of the infection. The results of our study suggest that the previous studies using in vitro methods involving soluble mucins or its components may have lead to erroneous conclusions about the in vivo interactions of $\boldsymbol{H}$ pylori and gastric mucus gel. The present findings argue against the hypothesis that degradation of gastric mucus by $H$ pylori is important in the pathogenesis of peptic ulcer.
\end{abstract}

(Gut 1995; 36: 327-329)

Keywords: Helicobacter pylori, duodenal ulcer, peptic ulcer, pathogenesis, mucus, viscosity.

It is well established that Helicobacter pylori, a Gram negative spiral bacillus, is present in nearly all patients with duodenal ulcer and in over $75 \%$ of patients with gastric ulcer. ${ }^{1} \mathrm{H}$ pylori is not an innocent bystander but plays a role in the pathogenesis of peptic ulcer as cure of the infection cures ulcer disease. ${ }^{2} 3$ The mechanism by which $H$ pylori contributes to the development of peptic ulcer, however, is unclear - one possibility is that $H$ pylori undermines the host defence mechanisms.

A number of studies have suggested that $H$ pylori can damage the protective mucus coat lining the gastric mucosa. ${ }^{4-7}$ Damage to the mucus gel might allow back-diffusion of noxious luminal contents such as acid and pepsin and result in tissue injury and ulcer formation. One supposition has been that the effectiveness of the mucus gel might be compromised if there were degradation of one of its constituents. These studies, however, suffered from a number of drawbacks that limit their interpretation in the in vivo situation. For example, the mucin used was often commercially obtained from animal sources and incubations with various fractions of $H$ pylori or media in which $H$ pylori was grown were performed. ${ }^{4}$ In addition, enrichment of the enzyme-containing fraction was often necessary to demonstrate a measurable effect. $^{7-9}$.

Natural mucus is a suspension of macromolecules (for example, mucoid substances, enzymes, shed cellular elements, etc) in a fluid matrix and should be studied fresh. Since neither the mucus (for example, soluble components) nor the experimental conditions used in previous reports even remotely reproduced the events taking place in natural intact human gastric mucus, we designed a study to assess the effects of $H$ pylori on gastric mucus gel viscosity. The study was patterned after a pre$H$ pylori study of human gastric mucus by Curt and Pringle. ${ }^{10}$

\section{Methods}

\section{PATIENTS}

Nineteen patients with endoscopically documented peptic ulcer disease participated in this study. Informed consent was obtained and the study was approved by the Institutional Review Board. The patients consisted of 18 men and one woman. Helicobacter pylori status was confirmed by the urea breath test as described previously. ${ }^{11}$ Infected patients were treated with a three drug regimen consisting of tetracycline (500 mg four times daily), metronidazole (250 $\mathrm{mg}$ three times daily), and tripotassium dicitrato-bismuthate (two tablets four times daily) given for two weeks. The infection was considered cured if the urea breath test was negative four or more weeks after completing the treatment.

Gastric secretions were collected in the morning after an overnight fast. A 18 Fr gastric sump nasogastric tube (Medovations Inc, Milwaukee, WI, USA) was introduced through the nose or mouth and positioned in the stomach. The position was checked by recovery of infused saline. The tube was connected to a low pressure, intermittent suction 


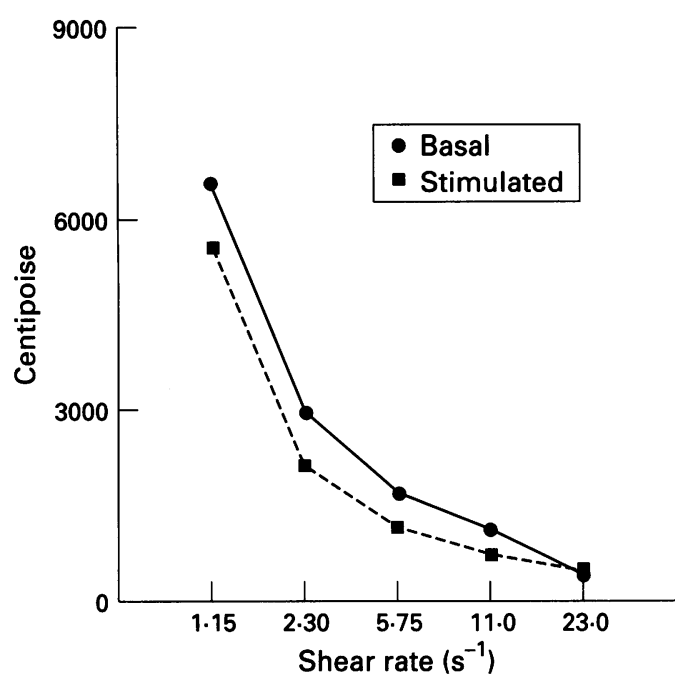

Figure 1: Results of mucus viscosity (in centipoise units) obtained in a single patient at different shear rates in inverse seconds $\left(s^{-1}\right)$. There was no statistical difference between the basal and pentagastrin stimulated samples at each sheer rate.

pump and the gastric secretions were collected in containers kept on ice. Four, 15 minute collections of basal secretions were obtained and pentagastrin $(6 \mu \mathrm{g} / \mathrm{kg})$ was then administered subcutaneously. Four additional 15 minute gastric secretions were obtained.

The samples were kept on ice during transfer to the laboratory and viscosity studies were carried out the same day as preliminary studies showed that freeze drying and storing the sample lead to falsely high mucus viscosity results (unpublished observation).

\section{VISCOSITY ANALYSIS}

The gastric juice specimens were centrifuged at $10000 \mathrm{rpm}$ for 15 minutes in a Sorvall SS-34 centrifuge. The supernatant was discarded and the insoluble mucus gel was used for the estimation of viscosity. ${ }^{10}$ Samples visually contaminated with food, bile, or blood were discarded. Briefly, the viscosity was measured in a rotational cone/plate microviscometer using a CP-52 spindle (Brookfield Engineering Laboratories Inc, Stoughton, MA, USA). The mucus gel $(0.5 \mathrm{ml})$ was placed in a sample cup and allowed to reach the thermostatically controlled temperature of $37^{\circ} \mathrm{C}$. The samples were exposed to 10 spindle rotations at each shear rate, varying from $1 \cdot 15 \mathrm{~s}^{-1}$ to $46 \cdot 0 \mathrm{~s}^{-1}$ and the viscosity (recorded as centipoise units) was measured at each shear rate.

\section{Results}

Twenty experiments were carried out on 19 patients; one patient had studies performed before and after cure of $H$ pylori infection. Gastric secretions obtained in seven experiments were contaminated with food debris, blood, or bile and were discarded. The remaining 13 were suitable for analysis and were included in the study: six from patients with active $H$ pylori infection and seven after eradication of the infection.

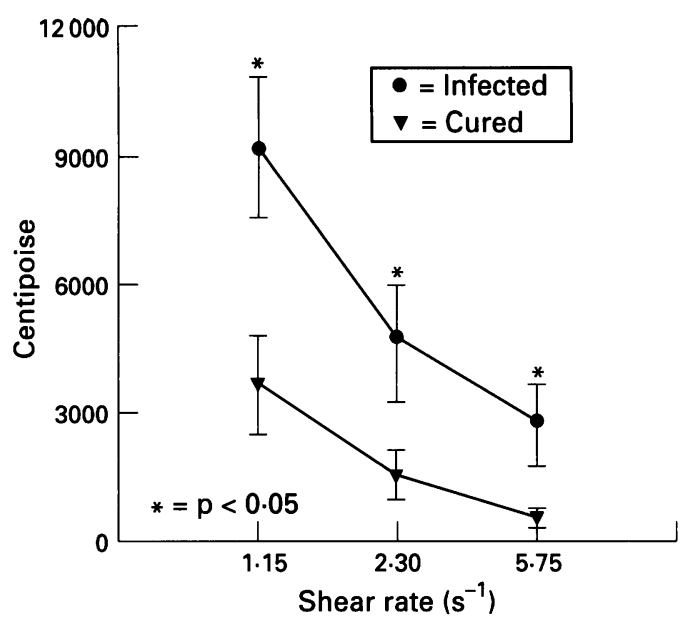

Figure 2: Gastric mucus viscosity in patients with and without infection with Helicobacter pylori. Each point indicates mean (SEM). The viscosity values were significantly higher in the infected group compared with the treated group at each shear rate. Mucus viscosity could not be measured at rates above $5 \cdot 75 \mathrm{~s}^{-1}$ in some samples as the values were beyond the measuring capacity of the viscometer.

At any single shear rate the viscosity of the samples was similar and at least one uncontaminated sample from each patient was available for analysis. There was no appreciable difference in the mucus viscosity of samples obtained under basal conditions and after pentagastrin stimulation (Fig 1). The results of basal and stimulated values were therefore combined. Viscosity measurements could be obtained from all patients at shear rates of $5.75 \mathrm{~s}^{-1}$ or less. Gastric mucus is a nonNewtonian substance in that its viscosity changes with changing rates of shear and at higher shear rates some samples had a viscosity that the viscometer could not measure. The gastric mucus viscosity was significantly higher in samples from patients infected with $H$ pylori than in mucus gel obtained after eradication of the infection (Fig 2).

\section{Discussion}

How $H$ pylori actually causes ulcers is poorly understood. One suggestion has been that $H$ pylori somehow weakens host protective factors, in particular gastric mucus. ${ }^{6} 12$ Mucus is a heterogeneous mixture of proteins, glycoproteins, and lipids. ${ }^{13}$ The complex structure of mucus glycoprotein polymer and the strong negative charge of the sulphated glycoproteins provides the mucus layer with visco-elastic, hydrophobic, and permselective properties. Mucus is an important lubricant and may also assist the mucosa in resisting the effects of gastric acid and pepsin. $H$ pylori lies both within and beneath the gastric mucus. ${ }^{14}$ It has been suggested that occupying the niche between the mucus layer and the mucosal cells allows the organism to damage both. ${ }^{12}$

Gastric mucus is a non-Newtonian substance in that its viscosity changes with changing rates of shear. By contrast, Newtonian substances such as water retain their viscosity irrespective of the sheet rate. Mucus has been described as a 'pseudoplastic material' since its viscosity decreases with increasing rates of 
shear. Viscosity is a measure of resistance to flow and is determined by the ratio of shear stress to shear rate. Therefore, the viscosity of non-Newtonian fluids must be measured by instruments such as the rotational cone-plate viscometer which measure both the shear stress and shear rate. We found that the insoluble mucus gel obtained from the stomachs of $H$ pylori infected individuals was more viscous than that obtained after $H$ pylori eradication.

In theory, $H$ pylori can damage the gastric mucus in a number of ways. For example, $H$ pylori proteases have been described that degrade mucus glycoproteins, and $H$ pylori produce at least two lipolytic enzymes, lipase and phospholipase A2. ${ }^{12}$ The lipolytic enzymes could degrade mucus lipids and result in a reduction in surface hydrophobicity as well as in formation of lysophospholipids. Lysophospholipids such as lysolecithins have potent lytic activity and could not only damage the mucus layer but also the cell membranes of the gastric epithelial cells. ${ }^{12} 15$

Although there is evidence that $H$ pylori can damage gastric mucin in vitro, these studies suffer from several potential limitations. The experiments were performed under in vitro conditions which did not attempt to duplicate the conditions in the human stomach. For example, the biochemical studies typically used purified, lyophilised, soluble porcine gastric mucin or purified components from soluble mucins. ${ }^{45}$ It is well established that the molecular structure and composition of mucus is very heterogeneous and important differences have been identified depending upon the species (pig, rat, human), site (stomach, small bowel, colon) and age (newborn $v$ adult) of the individual. ${ }^{13}$ Previous histological studies have shown gastric 'mucus depletion' in $H$ pylori infection. ${ }^{1617}$ The surface cells in gastritis have an increased turnover and are therefore younger and probably biologically and biochemically different to the cells lining the stomach of an uninfected person. Whether the apparent mucus depletion represents less mucus being made or increased release is not known with certainty. The results reported here may simply reflect the fact that, on average, younger gastric mucus cells produce a mucus that is biochemically different to that produced by the older cells from uninfected individuals.

To obviate the disadvantages of the in vitro studies, we examined directly the viscosity of fresh insoluble gastric mucus gel obtained from patients with and without $H$ pylori infection. Our experiments showed that the gastric mucus viscosity was higher in patients with $H$ pylori infection compared with that in the treated group. In a previous study, the gastric mucus viscosity of patients with duodenal ulcer disease was found to be higher than that in control subjects. ${ }^{10}$ The explanation of the difference between results remains unclear. The only obvious difference is that in the previous study, the $H$ pylori status of the patients was unknown. Perhaps there is a difference between gastric mucus gel viscosity between $H$ pylori gastritis and $H$ pylori duodenal ulcer.

The results of our study suggest that the previous studies using in vitro methods involving soluble mucus or its components may have lead to erroneous conclusions about the in vivo interactions of $H$ pylori and gastric mucus gel. The present findings argue against the hypothesis that degradation of gastric mucus by $H$ pylori is important in the pathogenesis of peptic ulcer.

This work was supported by the Department of Veterans Affairs and the generous support of Hilda Schwartz.

1 Peterson WL. Helicobacter pylori and peptic ulcer disease. N Engl f Med 1991; 324: 1043-8.

2 Graham DY, Lew GM, Klein PD, Evans DG, Evans DJ, Saeed ZA, et al. Effect of treatment of Helicobacter pylori infection on the long-term recurrence of gastric or duodenal ulcer: a randomized, controlled study. Ann Intern Med 1992; 116: 705-8.

3 Hentschel E, Brandstatter G, Dragosics B, Hirschl AM, Nemec $\mathrm{H}$, Schutze $\mathrm{K}$, et al. Effect of ranitidine and amoxicillin plus metronidazole on the eradication of Helicobacter pylori and the recurrence of duodenal ulcer. N Engl f Med 1993; 328: 308-12.

4 Slomiany BL, Bilski J, Sarosiek J, Murty VL, Dworkin B, VanHorn $\mathrm{K}$, et al. Campylobacter pylori degrades mucin and undermines gastric mucosal integrity. Biochem Biophys Res Commun 1987; 144: 307-14.

5 Sarosiek J, Slomiany A, Slomiany BL. Evidence of weakening of gastric mucus integrity by Campylobacter pylori. Scand f Gastroenterol 1988; 23: 585-90.

6 Ormond JE, Talley NL. Campylobacter pylori, mucus, and peptic ulceration. A dynamic interaction. $\mathfrak{f}$ Clin Gastroenterol 1989; 11: 492-5.

7 Slomiany BL, Murty VLN, Piotrowski J, Liau YH, Sundaram P, Slomiany A. Glycosulfatase activity of Helicobacter pylori toward gastric mucin. Biochem Biophysc Res Commun 1992; 183: 506-13.

8 Murty VLN, Piotrowski J, Morita M, Slomiany A, Slomiany BL. Inhibition of Helicobacter pylori glycosulfatase activity toward gastric sulfomucin by nitecapone. Biochem activity toward gastric

9 Slomiany BL, Murty VLN, Piotrowski J, Grabska M, Slomiany $\mathrm{A}$. Glycosulfatase activity of $\mathrm{H}$ pylori toward human gastric mucin: Effect of sucralfate. $A m, \tilde{J}$ Gastroenterol 1992; 87: 1132-7.

10 Curt JRN, Pringle R. Viscosity of gastric mucus in duodenal ulcer. Gut 1969; 10: 931-4.

11 Graham DY, Klein PD, Evans DJ Jr, Evans DG, Alpert LC, Operkun AR, et al. Campylobacter pylori detected noninvasively by the ${ }^{13} \mathrm{C}$-urea breath test. Lancet 1987; i: 1174-7.

12 Slomiany BL, Slomiany A. Mechanism of Helicobacter pylori pathogenesis: Focus on mucus. $\mathcal{f}$ Clin Gastroenterol pylori pathogenesis: Focus on
1992; 14 (suppl 1): S114-21.

13 Neutra MR, Forstner JF. Gastrointestinal mucus: synthesis, secretion and function. In: Johnson LR, ed. Physiology of the gastrointestinal tract, 2nd ed. New York: Raven Press, 1987: 975-1009.

14 Thomsen LL, Gavin JB, Tasman-Jones C. Relation of Helicobacter pylori to the human gastric mucosa in chronic gastritis of the antrum. Gut 1990; 31: 1230-6.

15 Slomiany BL, Sarosiek J, Liau YH, Laszewicz W, Slomiany A. Lysolecithin effects the viscosity, permeability and peptic susceptibility of gastric mucin. Scand $\mathcal{F}$ Gastroenterol 1986; 21: 1073-9.

16 Hui PK, Chan WY, Cheung PS, Chan JKC, Ng CS. Pathologic changes of gastric mucosa colonized by
Helicobacter pylori. Hum Pathol 1992; 23: 548-65.

17 Hansing RL, D'Amico H, Levy M, Guillan RA. Prediction of Helicobacter pylori in gastric specimens by inflammatory and morphological histological evaluation. Am $\mathcal{f}$ Gastroenterol 1992; 87: 1125-31. 